\title{
Pathologic complete response (pCR) rates and outcomes after neoadjuvant chemoradiotherapy with proton or photon radiation for adenocarcinomas of the esophagus and gastroesophageal junction
}

\author{
Cristina M. DeCesaris ${ }^{1}$, Melanie Berger ${ }^{2}$, J. Isabelle Choi ${ }^{3}$, Shamus R. Carr ${ }^{4}$, Whitney M. Burrows ${ }^{4}$, \\ William F. Regine ${ }^{5}$, Charles B. Simone $\mathrm{II}^{3}$, Jason K. Molitoris ${ }^{5}$ \\ ${ }^{1}$ Department of Radiation Oncology, University of Maryland Medical Center, Baltimore, MD, USA; ${ }^{2}$ University of Maryland School of Medicine, \\ Baltimore, MD, USA; ${ }^{3}$ New York Proton Center, New York, NY, USA; ${ }^{4}$ Department of Thoracic Surgery, University of Maryland Medical Center, \\ Baltimore, MD, USA; ${ }^{5}$ Department of Radiation Oncology, University of Maryland School of Medicine, Baltimore, MD, USA \\ Contributions: (I) Conception and design: CB Simone 2nd, JI Choi, SR Carr, WM Burrows, CM DeCesaris, JK Molitoris; (II) Administrative support: \\ CB Simone 2nd, JI Choi, JK Molitoris, WF Regine; (III) Provision of study materials or patients: CB Simone 2nd, JI Choi, JK Molitoris, WF \\ Regine, SR Carr, WM Burrows; (IV) Collection and assembly of data: CM DeCesaris, JK Molitoris, M Berger; (V) Data analysis and interpretation: \\ CM DeCesaris, M Berger, CB Simone 2nd, JI Choi, JK Molitoris, SR Carr, WM Burrows, WF Regine; (VI) Manuscript writing: All authors; (VII) \\ Final approval of manuscript: All authors. \\ Correspondence to: Cristina M. DeCesaris, MD. Department of Radiation Oncology, University of Maryland Medical Center, 22 S. Greene St., \\ Baltimore, MD 21201, USA. Email: cristina.decesaris@umm.edu.
}

Background: Pathologic complete response (pCR) after neoadjuvant chemoradiotherapy (nCRT) is associated with improved survival in patients treated for esophageal cancer. While proton beam therapy (PBT) has been demonstrated to reduce toxicities with nCRT, no data comparing pCR rates between modalities exist to date. We investigated pCR rates in patients with distal esophageal/GEJ adenocarcinomas undergoing trimodality therapy with nCRT-PBT or photon-based nCRT with the hypothesis that pathologic responses with PBT would be at least as high as with photon therapy.

Methods: A single-institutional review of patients with distal esophageal adenocarcinoma treated with trimodality therapy from 2015-2018 using PBT was completed. PBT patients were matched 1:2 to patients treated with photons. Chi square and two-sample $t$-tests were utilized to compare characteristics, and the Kaplan Meier method was used to estimate oncologic endpoints.

Results: Eighteen consecutive PBT patients were identified and compared to 36 photon patients. All patients received concurrent chemotherapy; $98 \%$ with carboplatin/paclitaxel. Most patients were male (91\%) and White (89\%); median age was 62 years (range, 31-76 years). Median radiation dose in both cohorts was 50.4 Gy (range, 41.4-50.4 Gy); all courses were delivered in 1.8Gy fractions. Age, gender and race were well balanced. Patients treated with PBT had a significantly higher pre-treatment nodal stage (N) and AJCC $7^{\text {th }}$ edition stage grouping $(\mathrm{P}=0.02, \mathrm{P}=0.03)$. Despite this, tumoral and nodal clearance and $\mathrm{pCR}$ rates were equivalent between cohorts $(\mathrm{P}=0.66, \mathrm{P}=0.11, \mathrm{P}=0.63$, respectively). Overall $\mathrm{pCR}$ and individual primary and nodal clearance rates, overall survival (OS), locoregional control (LRC), and distant metastatic control did not significantly differ between modalities (all $\mathrm{P}>0.05$ ). Major perioperative events were balanced; however, there were $5(14 \%)$ perioperative deaths in the photon cohort compared to $0(0 \%)$ in the proton cohort $(\mathrm{P}=0.06)$.

Conclusions: The use of PBT in trimodality therapy for distal esophageal adenocarcinoma yields pCR rates comparable to photon radiation and historical controls. Pathologic responses and oncologic outcomes in this study did not differ significantly between modalities despite PBT patients having higher AJCC stages and nodal disease burdens. 
Keywords: Esophageal neoplasms; radiotherapy; radiotherapy-conformal

Submitted Feb 10, 2020. Accepted for publication Jun 24, 2020.

doi: 10.21037/jgo-20-205

View this article at: http://dx.doi.org/10.21037/jgo-20-205

\section{Introduction}

Esophageal cancer portends a poor long-term prognosis, with 5 -year survival rates approaching only $20 \%$ (1). Despite poor long-term survival rates, approximately $50 \%$ of patients will initially present with local or locoregionalonly disease. Historical trials of surgery or radiation therapy alone for localized esophageal cancer yielded very limited 5-year survival rates of $0-6 \%(2,3)$, while early trials evaluating definitive chemoradiation (CRT) showed somewhat improved outcomes $(3,4)$. However, as rates of disease following CRT remained high (3), the addition of surgical resection was explored. Surgical resection followed by adjuvant chemotherapy and/or radiation therapy was felt to be suboptimal sequencing given high treatmentrelated morbidity and mortality, low rates of curative (gross, negative margin, "R0") resections with upfront surgery, and prolonged recovery periods delaying the start of adjuvant therapy (5-7), and thus focus began to shift towards neoadjuvant therapy followed by esophagectomy.

Over the last two decades, neoadjuvant chemoradiation (nCRT) followed by surgical resection has been established as the standard of care for locally advanced cancers of the esophagus and gastroesophageal junction (GEJ). Neoadjuvant chemotherapy (NAC) was not found to improve overall survival (OS) compared to surgery alone (8); however, early meta-analyses of combination neoadjuvant therapy demonstrated a survival benefit to nCRT compared to NAC or surgery alone $(9,10)$. The publication of the large, randomized "CROSS" trial in 2012 more definitively established combination nCRT followed by surgical resection, or "trimodality therapy" as standard of care by demonstrating improvements in R0 resections, pathologic response, and median and OS when nCRT was utilized (11).

With the increasing use of nCRT, pathologic response, and specifically pathologic complete response (pCR, clearance of both primary tumor and nodal disease) was determined to be a prognosticator for oncologic outcomes. Early studies demonstrated improvements in disease-free survival (DFS) and OS with pCR (12-14) and R0 resections $(12,13)$, and notably the CROSS trial showed that undergoing nCRT increased the likelihood of achieving both factors (11).

In recent years, the use of advanced radiation techniques (ARTs), including intensity-modulated radiation therapy (IMRT) and proton beam therapy (PBT) has increased steadily (15). Given its unique physical properties, namely its characteristic Bragg Peak, PBT in particular is an area of active research given its ability to minimize treatmentrelated toxicities for esophageal cancer (16).

Given the inherent differences between charged particles and $\mathrm{X}$-rays, protons and photons interact and deposit dose differently in matter. Protons are thought to have a relative biological effectiveness (RBE) to highenergy photons of 1.1; however, this is subject to great variability and is thought to possibly increase as high as 1.35 to 1.7 at the distal edge of spread-out Bragg Peaks (SOBP) (17). It is currently undefined how this increased biological effectiveness impacts tumor responses. In fact, pathologic tumoral responses to nCRT-PBT in comparison to photon-based nCRT have not been well characterized for numerous diseases, including esophageal/GEJ cancers. Given the prognostic implications of pCR on OS in esophageal/GEJ cancers, we investigated pCR rates in patients with esophageal/GEJ cancers, specifically distal adenocarcinomas, undergoing trimodality therapy with CRT-PBT or photon-based nCRT with the hypothesis that pathologic responses with PBT would be at least as high as with photon therapy. We present the following article in accordance with the STROBE reporting checklist (available at http://dx.doi.org/10.21037/jgo-20-205).

\section{Methods}

The research was conducted in accordance with the Declaration of Helsinki (as revised in 2013). The study was approved by the institutional review board of IRB organization 0000281 (Registration No.: IRB00000474), and informed consent was not required as this was a retrospective, de-identified, chart-review based study. A HIPAA compliant retrospective database was created of all consecutive patients treated for locally advanced esophageal 
or GEJ cancer who underwent trimodality therapy with nCRT-PBT or photon-based nCRT within our institution from 2015-2019. At our proton center, PBT is delivered via pencil-beam scanning, or "PBS". Eighteen nCRTPBT patients were identified and matched in a 1:2 fashion to 36 patients undergoing photon-based nCRT during a contemporary timeframe. All patients were matched for histology, tumoral location, and receipt of trimodality therapy, including a complete course of nCRT. It was decided to limit our population to adenocarcinoma only to control for potential differences in pathologic responses seen in other histologies, namely squamous cell carcinomas, and this in turn self-selected for more distally located tumors.

\section{Patient selection}

Inclusion criteria were a diagnosis of locally advanced esophageal or GEJ adenocarcinoma, defined as T2N0 (AJCC $7^{\text {th }}$ edition stage IIA) or higher, age $\geq 18$ years, receipt of full-course nCRT followed by definitive esophagectomy, and availability of pathology report for analysis. Patients were excluded if their treated disease represented a recurrent malignancy, for receipt of prior in-field radiation, if they did not complete an oncologic esophagectomy with nodal dissection. Within our study, "pCR" refers to patients with a pathologic staging of "pT0N0," indicating pCR in the resected esophagus and regional lymph nodes; "primary clearance" refers to a pCR in resected esophagus without concurrent nodal clearance, and conversely "nodal clearance" indicates pCR within the lymph node specimens without concurrent clearance of the resected esophageal primary.

\section{Determination of target volumes for radiation}

It is our institutional practice to delineate gross target volumes (GTV) from a combination of CT and PET-CT imaging, as well as endoscopic examination information. Patients undergo CT-simulation with IV contrast (when medically permissible) and esophageal paste, along with a 4D-CT to assess for respiratory-associated tumoral motion. The GTV is recontoured on all phases of the respiratory cycle. All patients undergo evaluation for the use of an abdominal compression belt, and at our proton facility voluntary breath-hold devices for respiratory-gated treatment delivery are available for tolerant patients. In the case of 4D-CT, the multi-phase GTVs are then combined to form an internal target volume (iGTV).

Our clinical target volume (CTV) is then generated by a GTV or iGTV expansion of 3-4 cm superiorly and inferiorly (unless constrained by the stomach, then by $2-3 \mathrm{~cm}$ ) and by an anatomically constrained $1 \mathrm{~cm}$ radial margin, as delineated by Wu et al., IFROBP 2015 (18). Gross nodal disease (GTVn) is delineated by CT/PET imaging and expanded by $1 \mathrm{~cm}$ to create a CTVn. Elective nodal coverage is per Wu et al., I7ROBP 2015 (18). Photon CTVs are expanded by $0.5 \mathrm{~cm}$ to obtain planning target volumes (PTVs) while proton plans are robustly optimized to account for beam-specific PTVs.

Dose is prescribed to the PTV for photon cases and CTV for photon cases, with a goal of covering $95 \%$ of the target structure with at least $95 \%$ of the prescription dose.

\section{Data collection and statistical analysis}

Demographic, tumor, treatment, and oncologic outcomes were collected and analyzed for all patients in a retrospective fashion. Acute toxicities were graded prospectively on a weekly basis by treating physicians, and perioperative events were retrospectively assessed through in-depth chart reviews. Toxicities were graded according to Common Terminology Criteria for Adverse Events (CTCAE) v5.0 (19). Similar acute toxicities were grouped together for analysis; nausea and vomiting were reported together, as were esophagitis and dysphagia. Similarly, anorexia, weight loss, and dehydration were grouped to form "hydration/nutrition." Hematologic toxicities represented any decrease on complete blood counts (CBCs) drawn weekly that necessitated a dose-decrease in or holding of weekly chemotherapy. Staging was based on endoscopic ultrasound (EUS) examinations, and CT and/ or PET-CT scans. Pathologic response was ascertained through detailed review of esophagectomy pathology reports with initial specimens evaluated by a specialized pathologist.

Chi square analysis and two-sample t-tests were utilized to compare patient and treatment characteristics for categorical and continuous variables, respectively. The Kaplan Meier method was used to estimate follow-up and oncologic endpoints, including locoregional control (LRC), distant control (DC), progression-free survival (PFS), and OS. The log-rank test was used to compare outcomes by modality for survival curves generated utilizing the KaplanMeier method. For local and distant recurrences, patients who were event-free were censored at the date of last oncologic follow-up; for OS, patients were censored at the date last known alive. Median follow-up was calculated 
utilizing the reverse Kaplan-Meier method.

\section{Results}

\section{Patient characteristics}

Fifty-two patients with a diagnosis of esophageal adenocarcinoma seen for treatment at our proton center were screened for inclusion; 18 met eligibility criteria. After matching in a 2:1 fashion, a total of 54 patients were included for analysis; 36 treated with photon RT and 18 treated with PBT. All lesions were adenocarcinomas; one lesion had mixed adenosquamous differentiation. All lesions were located in the mid/lower thoracic or abdominal esophagus, or within the GEJ. Patients were predominantly Caucasian and male; median age was 62 years (31-76 years), and well balanced between cohorts (Table 1). All patients underwent neoadjuvant treatment with concurrent chemotherapy; $53 / 54$ patients (98\%) were treated with carboplatin and paclitaxel-based chemotherapy; one patient was treated with cisplatin and 5-fluorouracil. The median RT dose was 50.4 Gy (41.4-50.4 years) and did not differ by modality. All patients were treated in once daily doses of $1.8 \mathrm{~Gy} /$ fraction (Table 1).

Pre-treatment primary tumor $(\mathrm{T})$ stage did not differ between cohorts, but patients treated with PBT had significantly higher nodal (N) stage and overall AJCC $7^{\text {th }}$ edition stage grouping. The difference in N-staging appeared to be driven by a larger proportion of PBT patient presenting with $\mathrm{N} 2$ disease (Table 1). One patient in each cohort presented with stage IVA disease; however, this was only by virtue of nodal disease present below the renal vessels, and these patients were managed definitively. Both patients underwent induction carboplatin and paclitaxelbased chemotherapy, and were recommended to proceed to nCRT + esophagectomy after exhibiting excellent responses on interim PET-CT scans (Table 1).

\section{Pathologic responses and oncologic outcomes}

Eleven of 54 patients (20\%) achieved a pCR; pCR rates did not differ by modality $(17 \%$ vs. $22 \%, \mathrm{P}=0.63)$. Fourteen of 54 patients (26\%) achieved primary tumoral clearance, and of the 28 patients who were node-positive prior to neoadjuvant therapy, 17 (61\%) achieved nodal clearance (Table 2). Additionally, 7 (27\%) of patients who were clinically N0 were diagnosed with occult nodal disease at the time of esophagectomy. Rates of primary (22\% vs. $28 \%$,
$\mathrm{P}=0.66)$ and nodal clearance (50\% vs. $69 \%, \mathrm{P}=0.11)$ did not differ by modality. On univariate analysis, pre-treatment T-stage, N-stage, CTV volume, and total RT dose were not associated with achievement of a pCR (Table 3). As all lesions were adenocarcinomas, all RT was given in $1.8 \mathrm{~Gy}$ fractions, and chemotherapy was highly homogeneous, these variables were not analyzed separately.

At a median follow-up of 25 months, $63 \%$ of patients remained alive; $13 \%$ were lost to follow up (1 proton and 6 photon patients). When stratified by modality, patients treated with photons had a significantly longer median follow-up (18 vs. 28 months, $\mathrm{P}=0.01$ ). There were 7 $(13 \%)$ and 13 (24\%) locoregional and distant recurrences, respectively, within the entire study population; rates did not differ by modality $(\mathrm{P}=0.78, \mathrm{P}=0.55)$ (Table 2). Eighteenmonth OS for the entire population was 66\% (95\% CI, $59 \%$ to $73 \%$ ); $83 \%$ (95\% CI, $71 \%$ to $95 \%$ ) vs. $59 \%$ ( $95 \%$ CI, $50 \%$ to $68 \%$ ) for PBT vs. photon RT, respectively. There was no difference in survival by modality on log-rank test $(\mathrm{P}=0.31)$ (Figure 1). Eighteen-month LRC was $93 \%$ (95\% CI, 89\% to $97 \%$ ), and was similar between modalities [ $94 \%$ ( $95 \%$ CI, $89 \%$ to $99 \%$ ) vs. $92 \%$ (95\% CI, $87 \%$ to 97\%), proton $v$ s. photon, log-rank $\mathrm{P}=0.59$ ] (Figure 2). Eighteen-month DC was $71 \%$ (95\% CI, $64 \%$ to $78 \%$ ), with no difference between modalities [79\% (95\% CI, 68\% to $90 \%$ ) vs. $72 \%$ ( $95 \%$ CI, $63 \%$ to $81 \%$ ) proton $v$ s. photon, $\log$-rank $\mathrm{P}=0.93$ ] (Figure 3). For patients achieving a pCR, 18 -month OS was $90 \%$ (95\% CI, $80 \%$ to $100 \%)$ and $\operatorname{did}$ not differ between cohorts $(\mathrm{P}=0.62)$ (Figure 4).

\section{Toxicities}

Overall, treatment was well tolerated in both cohorts. There were expected rates of Grade 2 (G2) acute toxicities, namely esophageal toxicities such as esophagitis and dysphagia. There was one G3 toxicity in the form of dehydration in the PBT cohort; there were no $\geq \mathrm{G} 4$ acute toxicities observed in either cohort (Table 4$)$. There were $4(22 \%)$ major perioperative events in the PBT cohort, and 7 (19\%) major perioperative events in the photon cohort; this did not differ statistically $(\mathrm{P}=0.81)$ (Table 5). In the photon cohort, 5 perioperative events led to death; no patients in the PBT arm expired from surgical complications; this trended towards significance in favor of $\mathrm{PBT}(\mathrm{P}=0.06)$. The majority of perioperative complications in both cohorts were in the form of anastomotic leaks $(\mathrm{n}=5)$; detailed descriptions of all perioperative events, including cause of death, were 
Table 1 Patient characteristics, analyzed using two-sample $t$-tests for continuous variables and Chi Square for categorical variables

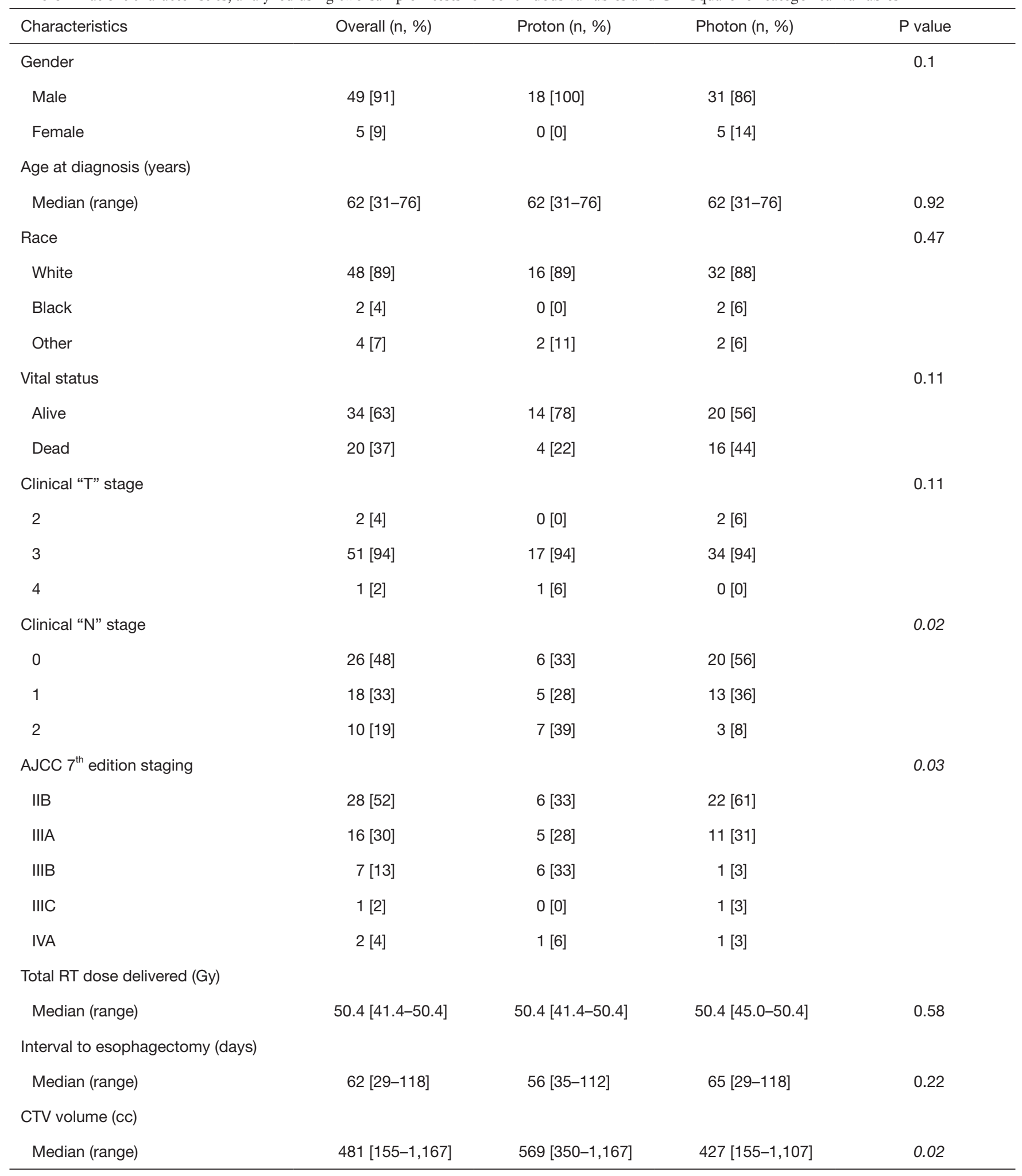

Significant values are in italic. 
Table 2 Pathologic responses and oncologic outcomes analyzed by Chi-Square

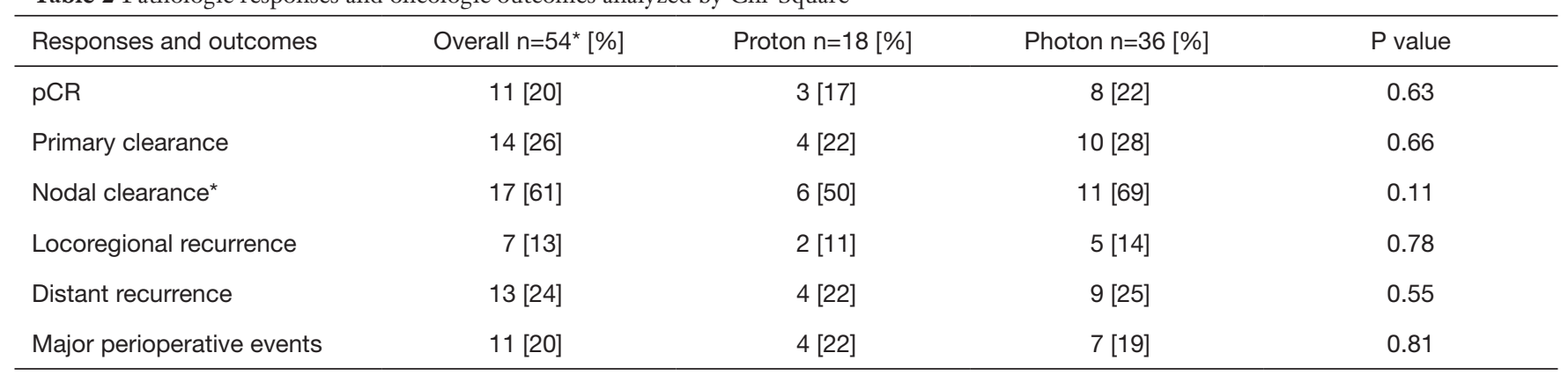

*, overall, $52 \%$ of patients (28 overall: 12 PBT and 16 photon) presented with nodal disease, and were evaluated for nodal clearance.

Table 3 Impact of pre-treatment oncologic factors on pCR rates, utilizing independent $t$-tests

\begin{tabular}{|c|c|c|c|c|}
\hline Characteristic & Overall $(n=54)$ & $\operatorname{pCR}(n=11)$ & No $p C R(n=43)$ & $P$ value \\
\hline t-stage [\%] & & & & 0.78 \\
\hline 1 & $0[0]$ & $0[0]$ & $0[0]$ & \\
\hline 2 & $2[4]$ & $0[0]$ & $2[5]$ & \\
\hline 3 & 51 [94] & $11[100]$ & $40[93]$ & \\
\hline 4 & $1[2]$ & $0[0]$ & $1[2]$ & \\
\hline n-stage [\%] & & & & 0.1 \\
\hline 0 & $26[48]$ & $7[64]$ & 19 [44] & \\
\hline 1 & 18 [33] & $4[36]$ & 14 [33] & \\
\hline 2 & $10[19]$ & $0[0]$ & $10[23]$ & \\
\hline \multicolumn{5}{|l|}{ CTV volume (cc) } \\
\hline Median [range] & $481[155-1,167]$ & $350[166-1,107]$ & $510[155-1,167]$ & 0.13 \\
\hline \multicolumn{5}{|l|}{ Total RT dose (Gy) } \\
\hline Median [range] & $50.4[41.4-50.4]$ & $50.4[45-50.4]$ & $50.4[41.4-50.4]$ & 0.27 \\
\hline \multicolumn{5}{|c|}{ Interval to surgery (days) } \\
\hline Median [range] & 62 [29-118] & 70 [54-84] & 59 [29-118] & 0.53 \\
\hline
\end{tabular}

provided in Table 5.

\section{Discussion}

In the first report comparing pCR rates between PBS PBT and photon therapy for patients with distal esophageal/ GEJ adenocarcinoma, we have found pCR rates to be equivalent between modalities, and congruous with historical adenocarcinoma photon controls (11). This was a single-institutional analysis of a homogenous group of patients undergoing standard trimodality therapy. A singlearm prospective study evaluating PBT using passively scattered protons as part of nCRT demonstrated overall pCR of $28 \%$; however, this study included squamous cell carcinoma patients $(23 \%)$ and did not include any direct comparison to patients treated with photon RT (20). Given prior studies establishing pCR as a prognostic factor for a number of oncologic outcomes in esophageal cancer (12-14), and the potential for an increased RBE exhibited by PBT, it is important to establish expected pCR rates with PBT, as well as quantifying potential differences from photon RT. The ability to demonstrate non-inferiority through such objective measures will become increasingly important as utilization of PBT, including PBS, continues to be 

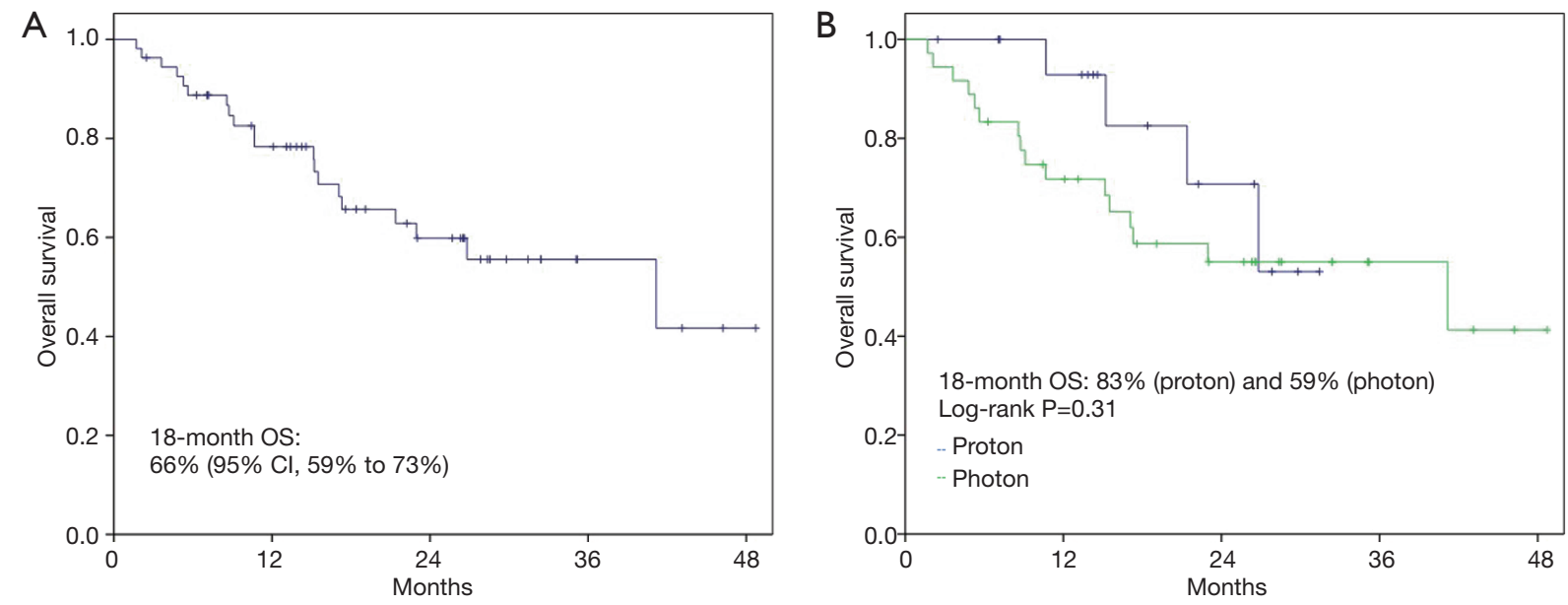

Figure 1 OS for all patients (A) and stratified by modality (B) by the Kaplan-Meier method and log-rank test. OS, overall survival.
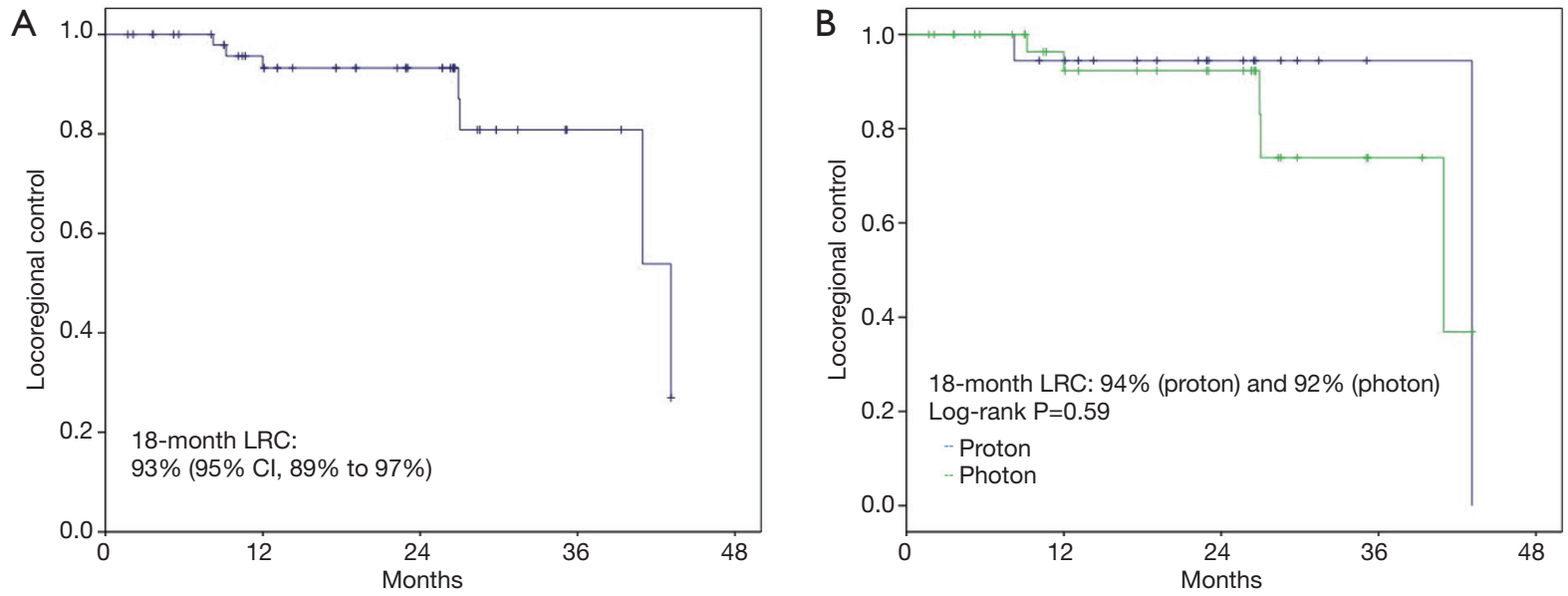

Figure 2 LRC for all patients (A) and stratified by modality (B) by the Kaplan-Meier method and log-rank test. LRC, locoregional control.
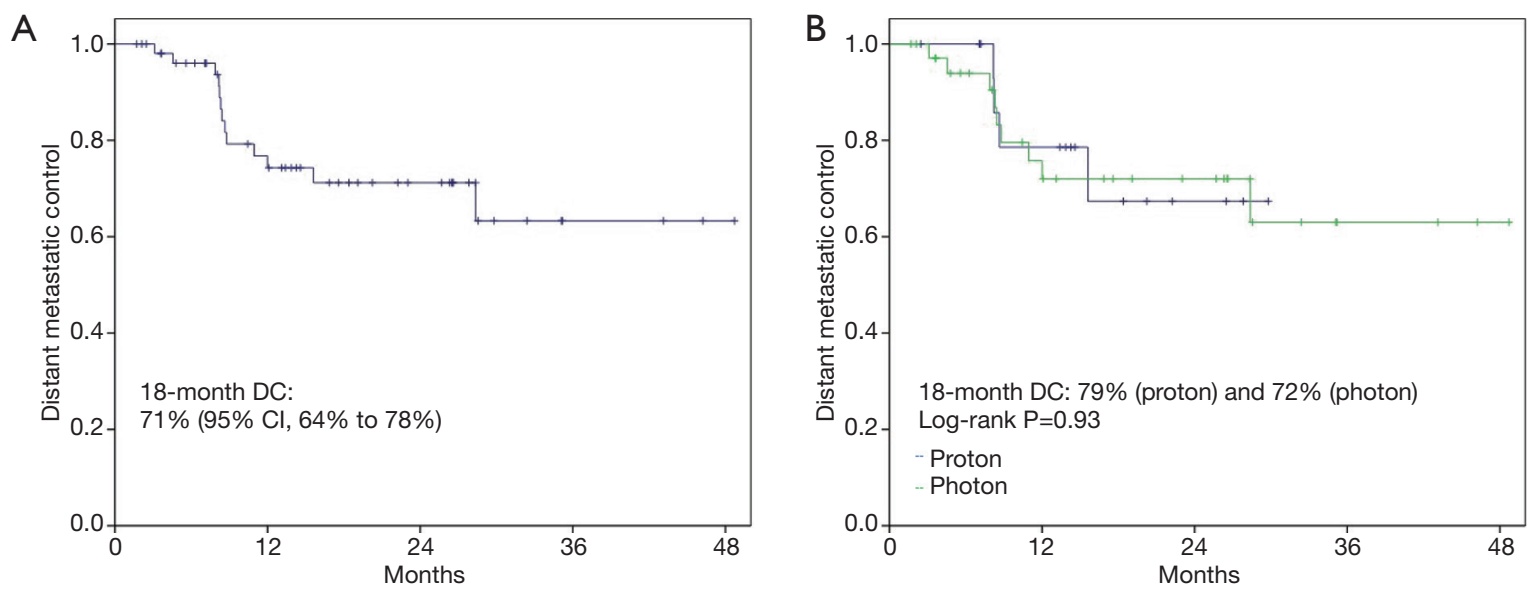

Figure 3 DC for all patients (A) and stratified by modality (B) by the Kaplan-Meier method and log-rank test. DC, distant control. 

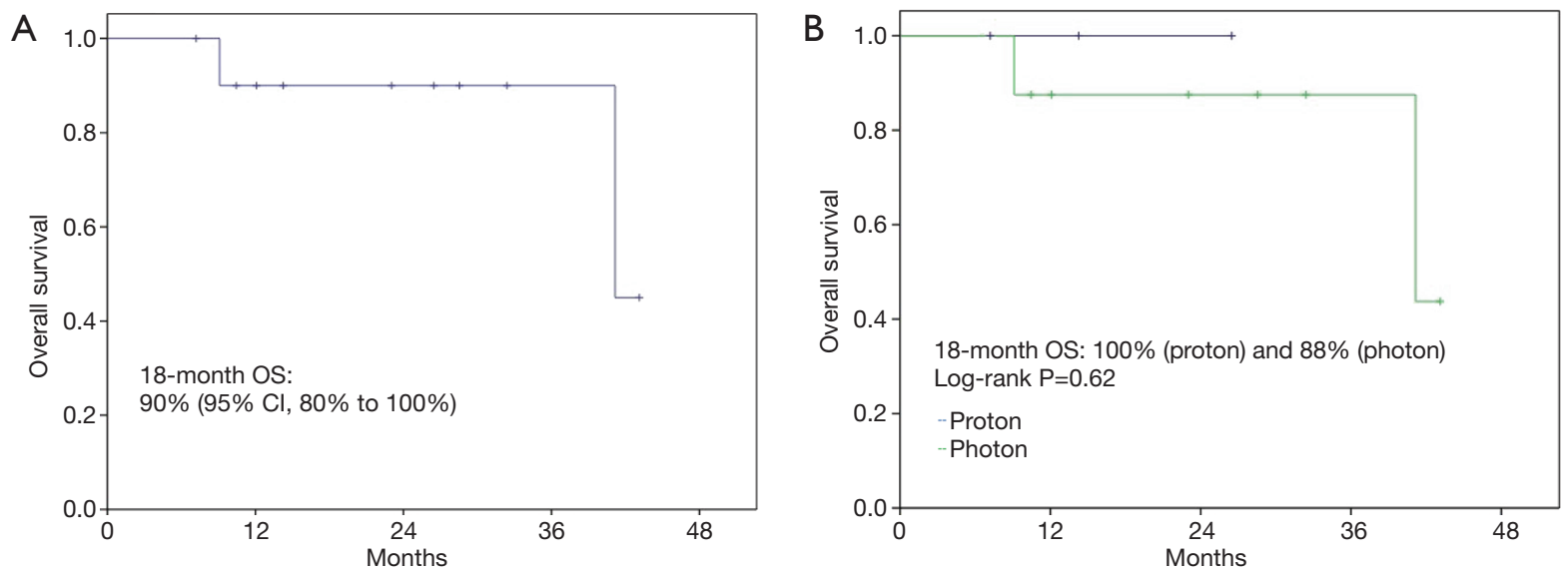

Figure 4 OS for patients achieving pCR, all patients (A) and stratified by modality (B) by the Kaplan-Meier method and log-rank test. OS, overall survival; pCR, pathologic complete response.

Table 4 Grade $\geq 2$ acute toxicities

\begin{tabular}{lcc}
\hline Toxicity & Proton $(n=18)[\%]$ & Photon $(n=36)[\%]$ \\
\hline Nausea/vomiting & $1[6]$ & $2[6]$ \\
Grade 2 & & \\
Esophagitis/ & & $10[28]$ \\
dysphagia & $6[33]$ & \\
Grade 2 & & \\
Fatigue & $0[6]$ \\
Grade 2 & & \\
Hydration/nutrition & & $7[19]$ \\
Grade 2 & $1[6]$ & $0[0]$ \\
Grade 3 & $1[6]$ & \\
Hematologic & & $6[17]$ \\
Grade 2 & $1[6]$ &
\end{tabular}

more frequently utilized. Our oncologic outcomes are in accordance with historical controls and also did not differ by modality.

PBT has the potential to reduce the risk of acute and late treatment-related toxicities, as well as surgical complications when applicable, thus improving the overall safety profile of radiation therapy in select esophageal/GEJ patients. In patients undergoing trimodality therapy for esophageal and GEJ cancers, larger retrospective series have demonstrated the positive impact of advanced radiation modalities, including PBT, to reduce post-operative toxicities (20-23); however, these studies did not include information regarding pathologic response rates. Similarly, the primary endpoint of the first prospective study investigating the use of neoadjuvant PBT for esophageal cancers was toxicitybased (23). Preliminary results of this randomized Phase II study comparing nCRT-PBT with nCRT-IMRT have been reported and demonstrate a decreased total toxicity burden (TTB) in favor of nCRT-PBT with comparable PFS, continuing to support the use of PBT in the neoadjuvant setting (23); however, there remains little reported information on potential differences in pathologic responses of esophageal cancers following exposure to various RT modalities.

Although the numbers of perioperative events were similar between our cohorts, it is worth noting that 5 of these events resulted in patient deaths in the photon cohort. There were 2 events within 30 days of surgery giving a similar rate as the CROSS study (5.6\% vs. $6 \%)$, along with 2 additional events 30-60 from surgery and one 7 months from surgery. There were no treatment-related or perioperative deaths in the PBT cohort. Although the instance of hepatic infarction due to aberrant vasculature is unlikely to be RT-related, this pattern of events is otherwise hypothesis-generating in that patients in the photon cohort appeared to have a more difficult time tolerating surgical complications, and is consistent with recent prospective data (23). This observed difference in perioperative mortality may translate to the trend towards improved OS we observed in the proton cohort.

It is important to note that similarities in pCR rates, as well as oncologic outcomes, existed despite the higher pretreatment nodal and AJCC staging of the PBT cohort. 
Table 5 Clinical descriptions of major perioperative events

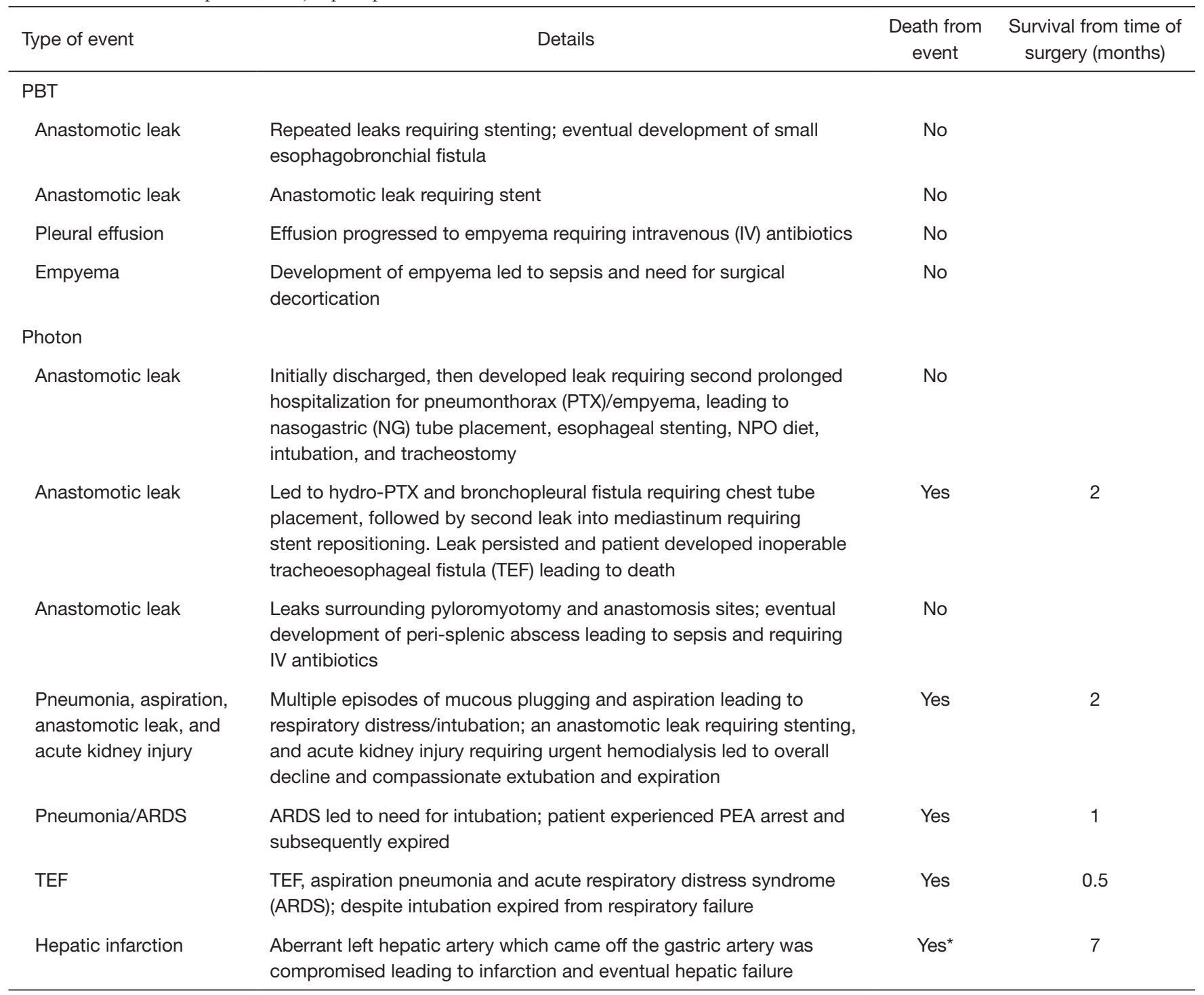

*, death was not in the immediate perioperative time period but was directly related to surgical complications.

The difference in staging may reflect a degree of inherent physician selection bias; often times, patients with a high nodal burden require very extensive RT fields, leading physicians to be more likely to recommend PBT to minimize the amount of integral RT exposure to critical thoracoabdominal tissues. In our study, nCRT-PBT was very well tolerated and suggested a more favorable acute toxicity profile in the areas of hematologic and nutritional side effects, although it is difficult to draw definitive conclusions given the small sample size and low overall number of toxicity events.

In our study, we chose to limit our selection criteria to distal adenocarcinomas located from the mid-thoracic esophagus through the GEJ for a number of reasons. We elected to analyze adenocarcinomas alone to control for any confounding variability in pathologic response that could arise through the inclusion of a heterogeneous group of histologies. Distal lesions tend to approximate a high number of critical OARs such as the heart, lung, liver, and stomach, resulting in the potential for greater amounts of radiation exposure to these areas, and within our academic medical center prompt referrals for PBT. The authors feel that this offers additional support for the ability of proton RT to improve acute toxicities and post-operative 
outcomes, given the favorable profiles demonstrated in our PBT cohort. Offering objective support for the observed improvements seen in the previously mentioned studies, dosimetric studies of proton RT for esophageal/GEJ cancers have objectively demonstrated decreased radiation exposure to critical thoracic organs such as the heart and lungs (24) as well as a decrease in severe lymphopenia $(25,26)$ when compared to photon RT.

Our study is limited by the typical challenges of retrospective research, although we mitigated this by utilizing an objective measure as our primary endpoint. We also attempted to mitigate confounding factors by limiting our study population to a relatively homogenous patient population of distal adenocarcinoma esophageal/ GEJ tumors. Additionally, all of the patients were treated within a single institution, limiting the potential for perioperative complications due to surgical expertise or perioperative care. Furthermore, we included all consecutive PBT patients and matched those with twice as many photon patients.

\section{Conclusions}

When treating locally advanced distal adenocarcinomas of the esophagus/GEJ with definitive trimodality therapy, there were no differences in primary, nodal, or pCR rates between patients treated with PBT or photon RT despite patients treated with PBT having significantly higher pretreatment nodal staging and AJCC stage grouping. Patients treated with nCRT-PBT had a very favorable acute toxicity profile, with potential improvements in perioperative mortality. Eighteen-month OS, LRC, and DC did not differ by modality. The use of PBT in trimodality therapy for this cohort of patients is safe and yields pCR rates comparable to photon RT and historical controls.

\section{Acknowledgments}

Funding: None.

\section{Footnote}

Reporting Checklist: The authors have completed the reporting checklist. Available at http://dx.doi.org/10.21037/ jgo-20-205

Conflicts of Interest: All authors have completed the ICMJE uniform disclosure form (available at: http://dx.doi. org/10.21037/jgo-20-205). The authors have no conflicts of interest to declare.

Ethical Statement: The authors are accountable for all aspects of the work in ensuring that questions related to the accuracy or integrity of any part of the work are appropriately investigated and resolved. The research was conducted in accordance with the Declaration of Helsinki (as revised in 2013). The study was approved by the institutional review board of IRB organization 0000281 (Registration No.: IRB00000474), and informed consent was not required as this was a retrospective, de-identified, chart-review based study.

Open Access Statement: This is an Open Access article distributed in accordance with the Creative Commons Attribution-NonCommercial-NoDerivs 4.0 International License (CC BY-NC-ND 4.0), which permits the noncommercial replication and distribution of the article with the strict proviso that no changes or edits are made and the original work is properly cited (including links to both the formal publication through the relevant DOI and the license). See: https://creativecommons.org/licenses/by-nc-nd/4.0/.

\section{References}

1. Howlader N, Noone AM, Krapcho M, et al. Esophageal Cancer - Cancer Stat Facts. SEER Cancer Statistics Review 2017 [cited 2019 Dec 3]. Available online: https:// seer.cancer.gov/statfacts/html/esoph.html

2. Earlam R, Cunha-Melo JR. Oesophogeal squamous cell carcinomas: II. A critical view of radiotherapy. Br J Surg 1980;67:457-61.

3. Cooper JS, Guo MD, Herskovic A, et al. Chemoradiotherapy of locally advanced esophageal cancer: long-term follow-up of a prospective randomized trial (RTOG 85-01). Radiation Therapy Oncology Group. JAMA 1999;281:1623-7.

4. Herskovic A, Martz K, al-Sarraf M, et al. Combined Chemotherapy and Radiotherapy Compared with Radiotherapy Alone in Patients with Cancer of the Esophagus. N Engl J Med 1992;326:1593-8.

5. Fok M, Sham JST, Choy D, et al. Postoperative radiotherapy for carcinoma of the esophagus: $\mathrm{A}$ prospective, randomized controlled study. Surgery 1993;113:138-47.

6. Zieren HU, Müller JM, Jacobi CA, et al. Adjuvant postoperative radiation therapy after curative resection 
of squamous cell carcinoma of the thoracic esophagus: A prospective randomized study. World J Surg 1995;19:444-9.

7. Ando N, Iizuka T, Ide H, et al. Surgery plus chemotherapy compared with surgery alone for localized squamous cell carcinoma of the thoracic esophagus: A Japan Clinical Oncology Group Study - JCOG9204. J Clin Oncol 2003;21:4592-6.

8. Kelsen DP, Ginsberg R, Pajak TF, et al. Chemotherapy followed by surgery compared with surgery alone for localized esophageal cancer. N Engl J Med 1998;339:1979-84.

9. Gebski V, Burmeister B, Smithers BM, et al. Survival benefits from neoadjuvant chemoradiotherapy or chemotherapy in oesophageal carcinoma: a meta-analysis. Lancet Oncol 2007;8:226-34.

10. Sjoquist KM, Burmeister BH, Smithers BM, et al. Survival after neoadjuvant chemotherapy or chemoradiotherapy for resectable oesophageal carcinoma: An updated metaanalysis. Lancet Oncol 2011;12:681-92.

11. van Hagen P, Hulshof MCCM, van Lanschot JJB, et al. Preoperative Chemoradiotherapy for Esophageal or Junctional Cancer. N Engl J Med 2012;366:2074-84.

12. Berger AC, Farma J, Scott WJ, et al. Complete response to neoadjuvant chemoradiotherapy in esophageal carcinoma is associated with significantly improved survival. J Clin Oncol 2005;23:4330-7.

13. Meredith KL, Weber JM, Turaga KK, et al. Pathologic response after neoadjuvant therapy is the major determinant of survival in patients with esophageal cancer. Ann Surg Oncol 2010;17:1159-67.

14. Reynolds JV, Muldoon C, Hollywood D, et al. Long-term outcomes following neoadjuvant chemoradiotherapy for esophageal cancer. Ann Surg 2007;245:707-16.

15. Haque $W$, Verma V, Butler EB, et al. Utilization of neoadjuvant intensity-modulated radiation therapy and proton beam therapy for esophageal cancer in the United States. J Gastrointest Oncol 2018;9:282-94.

16. Verma V, Lin SH, Simone CB, et al. Clinical outcomes and toxicities of proton radiotherapy for gastrointestinal neoplasms: a systematic review. J Gastrointest Oncol 2016;7:644-64.

17. Paganetti H. Relative biological effectiveness (RBE) values for proton beam therapy. Variations as a function of biological endpoint, dose, and linear energy transfer. Phys Med Biol 2014;59:R419-R472.
18. Wu AJ, Bosch WR, Chang DT, et al. Expert Consensus Contouring Guidelines for Intensity Modulated Radiation Therapy in Esophageal and Gastroesophageal Junction Cancer. Int J Radiat Oncol Biol Phys 2015;92:911-20.

19. CTCAE Files. 2018 [cited 2020 Jan 2]. Available online: https://evs.nci.nih.gov/ftp1/CTCAE/About.html

20. Lin SH, Komaki R, Liao Z, et al. Proton beam therapy and concurrent chemotherapy for esophageal cancer. Int J Radiat Oncol Biol Phys 2012;83:e345-e351.

21. Lin SH, Merrell KW, Shen J, et al. Multi-institutional analysis of radiation modality use and postoperative outcomes of neoadjuvant chemoradiation for esophageal cancer. Radiother Oncol 2017;123:376-81.

22. Wang J, Wei C, Tucker SL, et al. Predictors of postoperative complications after trimodality therapy for esophageal cancer. Int J Radiat Oncol Biol Phys 2013;86:885-91.

23. Lin SH, Hobbs B, Thall P, et al. Results of a phase II randomized trial of proton beam therapy vs intensity modulated radiation therapy in esophageal cancer. Int J Radiat Oncol 2019;105:680-1.

24. Shiraishi Y, Xu C, Yang J, et al. Dosimetric comparison to the heart and cardiac substructure in a large cohort of esophageal cancer patients treated with proton beam therapy or Intensity-modulated radiation therapy. Radiother Oncol 2017;125:48-54.

25. Shiraishi Y, Fang P, Xu C, et al. Severe lymphopenia during neoadjuvant chemoradiation for esophageal cancer: a propensity matched analysis of the relative risk of proton versus photon-based radiation therapy. Radiother Oncol 2018;128:154-60.

26. Fang P, Shiraishi Y, Verma V, et al. Lymphocyte-sparing effect of proton therapy in patients with esophageal cancer treated with definitive chemoradiation. Int J Part Ther 2018;4:23-32.

Cite this article as: DeCesaris CM, Berger M, Choi JI, Carr SR, Burrows WM, Regine WF, Simone CB 2nd, Molitoris JK. Pathologic complete response (pCR) rates and outcomes after neoadjuvant chemoradiotherapy with proton or photon radiation for adenocarcinomas of the esophagus and gastroesophageal junction. J Gastrointest Oncol 2020;11(4):663673. doi: 10.21037/jgo-20-205 